Reprinted from Journal of Mathematics and Physics, Massachusetts Institute of Technology, Vol. I, No.1.

\title{
THE EQUATION OF STATE, WITH APPLICATIONS TO VISCOSITY
}

\author{
By H. В. Phillips
}

1. For some years Prof. F. G. Keyes ${ }^{1}$ has used an equation of state of the form

$$
p=\frac{R T}{V-\beta e^{\frac{a}{v}}}-\frac{A}{(V-l)^{2}},
$$

where $p, V, T$ are pressure, volume, temperature, $R$ is the gas constant, and $\alpha, \beta, A, l$ are constants characteristic of the substance. This equation has been found to represent the pressure of a number of gases within the error of measurement provided the values of $V, T$ are not too near the saturation curve, and also to represent the pressure of liquids fairly well. It is expected to apply to fluids containing a single type of molecule over ranges of volume and temperature within which no changes of polymerisation or association occur. In this paper I give a general discussion of this type of equation and apply it to the calculation of viscosities.

2. A general type of system. The equation of Keyes, like that of van der Waals, belongs to the general type

$$
p=T \psi(V)-\phi(V),
$$

in which the pressure is a linear function of the temperature with coefficients that are functions of the volume. Substances for which this is true have other simple properties that entitle them to special consideration.

If $S$ is the entropy and $U$ the energy, (2) combined with the general thermodynamic equation

$$
d U=T d S-p d V
$$

gives

$$
d U=T[d S-\psi(V) d V]-\phi(V) d V .
$$

1A New Equation of Continuity, Proc. Nat. Acad. Science, 3(323-330). 
Since $d U$ and $\phi(V) d V$ are both exact differentials,

$$
T[d S-\psi(V) d V]
$$

must be exact. Hence

$$
d S-\psi(V) d V
$$

is the differential of a function of the temperature, and so

$$
S=\int \psi(V) d V+F(T)=\chi(V)+F(T)
$$

is the sum of a function of the temperature and a function of the volume. Conversely, if the entropy has this form, substitution in (3) gives

$$
d U=T F^{\prime}(T) d T+[T \psi(V)-p] d V
$$

Since the first two terms of this expression are exact differentials

$$
T \psi(V)-p
$$

must be a function of the volume and so $p$ has the form (2).

Equations (2) and (6) give

$$
U=\int T F^{\prime}(T) d T+\int \phi(V) d V=K(T)+P(V)
$$

showing that the energy is the sum of a function of the temperature and a function of the volume. Conversely, if the energy has this form, from (3) we have

$$
d S=F^{\prime}(T) d T+\frac{1}{T}[p+\phi(V)] d V,
$$

whence

$$
\frac{1}{T}[p+\phi(V)]
$$

is a function of the volume and so the pressure is given by an equation of the form (2).

From (7) the specific heat at constant volume is

$$
C_{\nu}=\frac{\partial U}{\partial T}=K^{\prime}(T)
$$

which is a function of the temperature only. Conversely, from (8) we find that the energy has the form (7) and so the pressure has the form (2).

From any one of the equations (2), (5), (7) (8) each of the others can then be obtained. If a substance has any one of these prop- 
erties it has all the others. That the pressure is a linear function of the temperature in case there is no change of association is indicated by such approximations as Charles' law and van der Waals' equation and by the experimental work of Amagat, ${ }^{2}$ Ramsey and Young. ${ }^{3}$ Keyes, and others. If the temperature is proportional to the average kinetic energy per degree of freedom, in case of invariable molecules the distribution in velocity will depend on the temperature only and that in configuration on the volume only, the probability of a given distribution will be the product of a function of the temperature and a function of the volume, and so the entropy

$$
S=K \log W
$$

will be the sum of such functions as indicated by (5). If the energy can be considered as the sum of two parts, the one kinetic due to the motion of the molecules, the other potential due to their relative positions, the first from kinetic theory (even in case of quantum theory) is a function of the temperature and the second a function of the volume. Hence the energy has the form (7). The specific heat at constant volume is very difficult to measure. The variation with volume that has been obtained is very small and that may be accounted for by changes of molecular association. It seems therefore reasonable to assume that the properties (2), (5), (7), (8) are satisfied by a substance in such changes of state as do not involve a change in the character of its molecules.

3. The Gibbs surface. The state of a system consisting of one component is determined by two variables. Three variables are then connected by an equation. Gibbs showed that the equation

$$
U=f(S, V)
$$

connecting energy, entropy, and volume is a fundamental equation, ${ }^{4}$ in the sense that from it by differentiation and algebraic processes all the thermodynamic variables can be obtained.

2 Ann. chim. phys., 29 (505).

3 Zeit. fur phys. Chem. 1 (440).

Scientific Papers, I (86). 
Taking $U, S, V$ as the coördinates of a point in three dimensions, equation (9) represents a surface called the Gibbs surface.

In the case just discussed this surface has a particularly simple form. The equations

$$
\left\{\begin{array}{c}
U=K(T)+P(V) \\
S=F(T)+\chi(V)
\end{array}\right\}
$$

show that if $T$ is kept constant, the curve obtained by varying $V$ has the same form whatever constant value of $T$ is used. The isothermals on the Gibbs surface are therefore all alike. Any one can be moved into any other by a displacement parallel to the US-plane. Similarly the constant volume lines are all alike. The surface, called a translation surface, can be generated by the motion (without rotation) of an isothermal or an isochore.

If then we determine the values of the energy and entropy along one isothermal and one isochore, we can construct the surface by moving the isothermal along the isochore and so determine the thermodynamic properties of the substance at all other volumes and temperatures for which the equations (10) are valid.

4. The kinetic pressure. To obtain the functions $\psi(V)$ and $\phi(V)$ in equation (2) it is necessary to have some hypothesis as to their origin. We assume that

$$
T \psi(V)
$$

is the kinetic pressure due to the bombardment of the molecules at a point within the fluid. The external pressure $p$ is less than this by the cohesive pressure $\phi(v)$ due to the mutual potential energy of the molecules.

Let $m$ be the mass of a molecule, $u$ its x-component of velocity, $\nu$ the number of molecules per unit volume, and

$$
\nu f(u) d u
$$

the number of these with components of velucity between $u$ and $u+d u$. If the molecules did not interfere with each other, the number of these that would cross unit area of the $x y$-plane in unit time would be

$$
\nu u f(u) d u
$$


and the $\mathrm{x}$-component of momentum transferred would be

$$
\nu m u^{2} f(u) d u
$$

This is equivalent to a pressure over this area equal to

$$
\int_{-\infty}^{\infty} \nu m u^{2} f(u) d u=\nu \text { av. }\left(m u^{2}\right)=\rho R T,
$$

where $\rho$ is the density.

Equation (11) gives the pressure of a perfect gas. It is obtained on the assumption that each molecule describes its path uninfluenced by the others. Any interaction of the molecules will increase this pressure, for the distribution of velocities is assumed to be the same in any case. Interaction of the molecules will increase their migration and so increase the transfer of momentum, or pressure.

Consider a thin section of the fluid between parallel planes perpendicular to the $\mathrm{x}$-axis. By observing the molecules cross unit area of one of these planes we obtain a rate of transfer of momentum $I$ equal to the pressure. By observing the molecules between the two planes at a particular instant, and dividing them into two currents in opposite directions, we obtain the total rate of transfer (11). The two results are not the same. For the brief time $d t$ we must observe the molecules to obtain $I$ must be long enough for a large number of molecules to cross so that we may have an average result. Even in this brief time a fraction $R I d t$ of the total transfer $I d t$ will be reflected back. This reflection will occur across the boundary equally in both directions and so will not affect the distribution between the planes but will be counted twice by the observer at a particular boundary. Therefore the difference

$$
I-R I=\rho R T
$$

will be the rate of transfer observed between the planes at a particular instant of time.

Since the molecules themselves are the elementary reflectors, if the density is very small so that they may be supposed to act independently, the percentage of $I$ reflected will be proportional to the density and so

$$
R=b \rho
$$


where $b$ is constant. From (12) the kinetic pressure is then

$$
I=\frac{\rho R T}{1-b \rho}=\frac{R T}{V-b}
$$

as given by van der Waals' equation. In this the quantity $b$ has the dimensions of a volume but is not here regarded as connected in any way with the volume of the molecules. In fact, if the molectles influence each other, the quantity $b$ will exist even if the volume occupied by the molecules is indefinitely small.

The co-volume $b$ is a measure of the reflecting power per molecule. At higher densities this action of a molecule may be interfered with by other molecules. If this effect is additive, we will have

$$
d b=-a b d \rho
$$

where $\alpha$ is constant, as the change produced by an increase $d \rho$ in the density. Integration of this gives

$$
b=\beta e^{-a \rho}=\beta e^{-\frac{a}{v}}
$$

as in the equation of Keyes.

5. The cohesive pressure. The mutual potential energy of two molecules is a function of their distance and relative orientation. In an infinite mass of a fluid the number of molecules at each distance from a given one and having with respect to it a given orientation is proportional to the density. The same is true of a given number of molecules. The potential energy of a gram of the fluid is then

$$
P=A \rho=\frac{A}{V}
$$

where $A$ is constant. This is true whatever be the law of force between the molecules, provided merely that the law is independent of the density and that the force diminish at great distances sufficiently for the integrals with infinite limits to converge. Since atoms of electronic model are polarized, it may be that two atoms attract when oriented in certain ways and repel when 
oriented in others. Equation (16) is still valid if the distribution of orientations is independent of the density.

If the force between molecules is appreciable only at very short distances, equation (16) is valid for a mass of fluid in a finite domain, for the molecules within the fluid have the same potential energy as before and the number near the surface is not great enough appreciably to affect the result. That such is the case is evident since the pressure depends only on the volume and not on the shape of the containing vessel. The cohesive pressure is then

$$
\phi=-\frac{\partial P}{\partial V}=\frac{A}{V^{2}}
$$

as given by van der Waals.

Now a study of pressure data shows that the cohesive pressure of gases is represented better by an expression of the form

$$
\phi=\frac{A}{(V+l)^{2}}
$$

than by (17). This indicates that the force between two molecules is not determined simply by their mutual positions but changes with the density. One way of explaining this is by the variation in the dielectric constant. In a dielectric of inductivity $k$, the force between given charges is equal to the force that would exist if the dielectric were air divided by $k$. If then the molecules can be treated as substantially invariable electrical systems, all the forces which determine the cohesive pressure are reduced in this same ratio and so (17) must be replaced by

$$
\phi=\frac{A}{k V^{2}} \text {. }
$$

According to the theory of Lorentz, ${ }^{5}$ in case of a gas

$$
\frac{k-1}{k+2}=C \rho
$$

where $C$ is constant. Solving this for $k$ and substituting in (18) we get

$$
\phi=\frac{A(V-C)}{V^{2}(V+2 C)}=\frac{A}{V^{2}}\left(1-\frac{3 C}{V} \ldots\right) .
$$

5 Richardson, Electron Theory of Matter, p. 73. 
Since the ratio of $C$ to $V$ is usually very small, within the precision of the data, this is equal to

$$
\frac{A}{(V+l)^{2}}=\frac{A}{V^{2}}\left(1-\frac{2 l}{V} \ldots\right)
$$

if we take

$$
l=3 / 2 C .
$$

From pressure measurements it is very difficult to determine the quantity $l$. It is determined graphically as the intercept on a coördinate axis of the line obtained by using $V$ and

$$
\frac{1}{\sqrt{\phi}}
$$

as coördinates. A small error of slope may change its value greatly. In some cases it even has the wrong algebraic sign. Recent work on methane gives

$$
l=.566
$$

with a precision of perhaps a few per cent. Using the value

$$
k=1.00095
$$

at $0^{\circ} \mathrm{C}$. and 1 atmosphere, we get

$$
C=.41 \quad 3 / 2 C=.61
$$

which agrees with the measured value of $l$ as well as could be expected.

6. The viscosity of a gas. Let $u_{\circ}$ be the $x$-component of the velocity of the gas (mass motion) at the point $(x, y, z)$. Let the gas flow parallel to the $x$-axis in such a way that

$$
\frac{\partial u_{\circ}}{\partial z}=1
$$

Under these conditions the viscosity $\eta$ of the gas is defined as the total momentum transferred across tunit area of the xy-plane in unit time. To find the viscosity we therefore find the number of molecules with each velocity crossing unit area and multiplying in each case by the average $x$-momentum.

Let $\nu$ be the number of molecules per cubic centimeter and $w$ the z-component of the velocity of a molectule. As in $\$ 4$, if the 
molecules did not interfere with each other, the number with velocity components between $w$ and $w+d w$ that would cross unit area of the xy-plane in unit time would be

$$
\nu w f(w) d w
$$

But because of interference the number that do cross is

$$
\frac{\nu w}{1-b \rho} f(w) d w=\frac{\rho}{1-b \rho} \frac{w}{m} f(w) d w .
$$

Tracing the motion of a given molecule from the xy-plane backward in its course, the distance from that plane at which it probably had a given interaction with other molecules is inversely proportional to the density. The average momentum acquired in any such interaction is proportional to the reflection coefficient $b$. Therefore in a field of unit velocity gradient (21) the average momentum parallel to the $\mathrm{x}$-axis carried by a molecule of velocity component $w$ has the form

$$
\frac{b}{\rho} F(w) \text {. }
$$

Combining (22) and (23) we obtain for the total transfer of $\mathrm{x}$-momentum across unit area the expression

$$
\eta=\frac{b}{1-b \rho} \int_{\circ}^{\infty} \frac{w}{m} f(w) F(w) d w=\frac{b K}{1-b \rho}
$$

where $K$ is a function of the temperature only.

In the following table are given measured viscosities together with the calculated values of

$$
K=\eta \frac{(1-b \rho)}{b}
$$

From Amagat's pressure measurements Keyes ${ }^{6}$ finds for carbon dioxide

$$
\log b=.4650-\frac{.7065}{V}
$$

Inspection of the table shows that at each temperature the variations in $K$ show no particular trend for densities less than about 0.2. At densities greater than this (25) ceases to hold satisfactorily.

6 J. Am. Soc. Ref. Eng. 3 (7). 
Viscosity of Carbon Dioxide ${ }^{7}$ - Gas Phase

$\begin{array}{rrlccr}T & p & \rho & \log b & \eta \times 10^{6} & K \times 10^{8} \\ 20^{\circ} & 1 & .00183 & .4637 & 148 & 5.07 \\ & 20 & .036 & .4383 & 156 & 5.12 \\ & 40 & .100 & .3944 & 166 & 5.03 \\ & 50 & .145 & .3626 & 177 & 5.11 \\ & 56 & .190 & .3308 & 186 & 5.15\end{array}$

$\begin{array}{rrllll}30^{\circ} & 1 & .00177 & .4637 & 153 & 5.23 \\ & 20 & .0354 & .4400 & 159 & 5.21 \\ & 40 & .092 & .4000 & 168 & 5.14 \\ 60 & .177 & .3400 & 187 & 5.24 \\ & 70 & .287 & .2622 & 299 & 5.95\end{array}$

$\begin{array}{ccllll}32^{\circ} & 1 & .00176 & .4638 & 155 & 5.30 \\ & 20 & .0352 & .4401 & 162 & 5.31 \\ & 40 & .090 & .4014 & 175 & 5.36 \\ 60 & .170 & .3449 & 187 & 5.27 \\ & 70 & .255 & .2848 & 214 & 5.65 \\ 74 & .360 & .3107 & 254 & 6.49\end{array}$

$\begin{array}{rrllll}35^{\circ} & 1 & .00174 & .4638 & 156 & 5.33 \\ & 20 & .0348 & .4404 & 163 & 5.35 \\ & 40 & .085 & .4015 & 174 & 5.42 \\ 60 & .163 & .3498 & 178 & 5.05 \\ & 70 & .227 & .3046 & 214 & 5.75 \\ 75 & .289 & .2608 & 237 & 6.15\end{array}$

$\begin{array}{clllll}40^{\circ} & 1 & .00173 & .4638 & 157 & 5.37 \\ & 23.8 & .0408 & .4362 & 169 & 5.50 \\ 40 & .083 & .4064 & 176 & 5.34 \\ & 60 & .153 & .3569 & 187 & 5.43 \\ & 70 & .204 & .3209 & 200 & 5.47 \\ & 80 & .291 & .2594 & 218 & 5.65\end{array}$

7 Phillips, Proc. R. Soc. 87 (46). 
7. Vicosity of liquids. In the case of a liquid I assume that the molecules vibrate about positions of equilibrium as in a solid. Due to the mass motion of the fluid these equilibrium positions change with the time, but similar changes occur at neighboring points so that the structure of the liquid near a given molecule varies slowly in comparison with the motion of the molecule. This motion is therefore substantially periodic and so may be expected to obey the laws of quantum theory.

We may consider the motion of a molecule in two ways both leading to the same formula for viscosity. First we may assume the time of interaction of two molecules as very brief followed by a much longer period of practically uniform motion and that the velocity relative to the fluid is acquired during the brief interaction. Suppose then a molecule reaching the xy-plane has been moving for a time $t$ since the last collision. From (21) its momentum parallel to the $x$-axis will on the average be

$$
\text { mwt, }
$$

where $w$ is the velocity perpendicular to the xy-plane. Since $w$ is constant over practically the entire periud

$$
m w t=\frac{1}{w} \int_{0}^{t} m w^{2} d t .
$$

The molecule in the time $t$ will on the average describe a complete mean free path, that is, a half period in the sense of quantum theory. According to Sommerfeld's theory we therefore have

$$
2 \int_{0}^{t} m w^{2} d t=n h
$$

where $n$ is an integer. Consequently the average $x$-momentum transferred is

$$
m w t=\frac{n h}{2 w}
$$

From (22) the viscosity is then

$$
\eta=\frac{n h \rho}{2 m(1-b \rho)} \int_{-\infty}^{\infty} f(w) d w=\frac{n h \rho}{2 m(1-b \rho)}=\frac{n N h}{2 M(V-b)},
$$


where $N$ is the number of molecules per mol and $M$ the molecular weight of the substance.

A second method is to consider the action as extending along the whole path of the molecule. At the distance $z$ from the xyplane the average velocity is equal to the mass motion of the fluid which by (21) may be taken as $z$. The molecule in moving through the fluid may be considered as subject to a sort of drag proportional on the average to the velocity of the fluid. Molecules reaching the xy-plane with velocity $w$ may then be considered as deriving this velocity at the average distance

$$
\bar{z}=\frac{\int z d w}{\int d w}=\frac{z w \mid \begin{array}{c}
z=0 \\
w=0
\end{array}-\int w d z}{\int d w}=\frac{\int w^{2} d t}{w}
$$

and so have an average momentum equal to

$$
m \bar{z}=\frac{\int m w^{2} d t}{w}=\frac{n h}{2 w}
$$

as in (27). The viscosity is then given by (28) as before.

A comparison of (28) with experiment is given in an article now being published in the Proceedings of the National Academy of Sciences. In all cases it turns out that $n=6$ so that (28) takes the form

$$
\eta(V-b)=\frac{3 N}{M} h .
$$

It is to be noted that $3 N / M$ is the number of translational degrees of freedom in the volume $V$ of the liquid. This suggests that the equation might be derived by a more general procedure using the gram of fluid as the elementary mechanical system. 